05

\title{
Исследование плазменных колебаний в стеклообразных диэлектриках методом полного внешнего отражения рентгеновских лучей
}

\section{(C) В.М. Стожаров}

Российский государственный педагогический университет им. А.И. Герцена, 191186 Санкт-Петербург, Россия

e-mail: gut1111@yandex.ru

Поступило в Редакцию 28 ноября 2019 г.

В окончательной редакции 28 ноября 2019 г.

Принято к публикации 30 января 2020 г.

\begin{abstract}
Исследованы стеклообразные диэлектрики методами дисперсии плазмонов и асимметрии числа локализованных электронов в зоне формирования полного внешнего отражения рентгеновских лучей и возбуждения плазменных колебаний. Определены внутренние механические напряжения и связанные с ними поляризации исследованных диэлектриков. Выяснено отсутствие внутреннего механического напряжения в монокристалле фторида лития, а наблюдаемая поляризация вызвана большим макроскопическим дипольным моментом в этом монокристалле.
\end{abstract}

Ключевые слова: дисперсия, асимметрия, поляризация, монокристалл.

DOI: 10.21883/JTF.2020.07.49444.382-19

\section{Введение}

Согласно современным представлениям, классическая функция Блоха, описывающая состояние электрона в периодических структурах, заменяется в аморфных твердых телах волновыми функциями в приближении сильной связи [1,2]. Электроны, принимающие участие в плазменных колебаниях, характеризуются малыми пробегами, соизмеримыми с межатомным расстоянием в твердых телах. Концепция локализованных электронных состояний в аморфных телах, теоретически разработанная в работах Мотта и Дэвиса [2], подтверждена экспериментально для аморфного плавленого кварца методом спектроскопии в ультрамягкой области рентгеновского спектра [3-5]. В ряде работ показана возможность возбуждения в диэлектриках объемных и поверхностных плазмонов в оптическом и радиочастотном диапазонах [6-8]. Дисперсия плазмонов есть основа понимания роли электромагнитных волн в возбуждении плазменных колебаний в твердых телах. В известных работах [8-11] дисперсию плазмонов в металлах рассчитывали теоретически преимущественно на основе теории Друде для свободных электронов. Ранее мы исследовали особенности полного внешнего отражения (ПВО) рентгеновских лучей и возбуждения плазменных колебаний в металлах [12-14]. В настоящей работе приведены результаты изучения методом ПВО дисперсии плазмонов в стеклообразных диэлектриках.

\section{1. Методика измерения дисперсии плазмонов}

Дисперсия плазмонов представляется зависимостью их энергии $E_{P}$, измеренной при разных азимутальных углах $\beta$, от волнового вектора. В [10], где исследовалась дисперсия поверхностных плазмонов в структурах с проводящей пленкой, введено плазменное волновое число:

$$
k_{P}=\frac{\omega_{P}}{c}=\frac{E_{P}}{\hbar c} .
$$

Здесь $E_{P}-$ энергия плазмонов в электронвольтах $[\mathrm{eV}]$, измеряемая при азимутальном угле $\beta=0^{\circ}$ методом, описанным в $[14]\left(\hbar=6.577 \cdot 10^{-16} \mathrm{eV} \cdot \mathrm{s}\right.$ постоянная Планка, $c=3 \cdot 10^{8} \mathrm{~m} / \mathrm{s}$ - скорость света). Подставляя значения постоянных в соотношение (1) и учитывая повороты образца в его плоскости по азимутальному углу $\beta$ в пределах от 0 до $360^{\circ}$ с шагом $22.5^{\circ}$, получаем формулу для расчета плазменного волнового вектора в общем виде:

$$
\mathbf{k}_{P}=5.06 \cdot 10^{16} E_{P}(\mathrm{eV}) \cos \beta \AA^{-1} .
$$

\section{2. Расчеты микроскопических характеристик стеклообразных диэлектриков}

Рассчитаем микроскопические характеристики для плавленого кварца $\mathrm{SiO}_{2}$.

\section{1. Расчет зоны выхода $h$ рентгеновских лучей, испытавших ПВО и возбуждающих в плавленом кварце плазменные колебания локализованных электронов}

В справочнике [15] приведены массовые коэффициенты поглощения $\frac{\mu}{\rho}$ рентгеновских лучей для всех элементов таблицы Менделеева и для разных длин 
волн рентгеновского излучения. Для излучения $\mathrm{Cu} K_{\alpha_{1}}$ с длиной волны $\lambda=1.5406 \AA$ находим

$$
\left(\frac{\mu}{\rho}\right)_{\mathrm{Si}}=62.1 \mathrm{~cm}^{2} / \mathrm{g}\left(\frac{\mu}{\rho}\right)_{\mathrm{O}}=11.3 \mathrm{~cm}^{2} / \mathrm{g} .
$$

Для плавленого кварца общий массовый коэффициент поглощения рентгеновских лучей определяется соотношением

$$
\left(\frac{\mu}{\rho}\right)_{\mathrm{SiO}_{2}}=\frac{M_{\mathrm{Si}}}{M}\left(\frac{\mu}{\rho}\right)_{\mathrm{Si}}+\frac{2 M_{\mathrm{O}}}{M}\left(\frac{\mu}{\rho}\right)_{\mathrm{O}},
$$

где молярная масса $M$ плавленого кварца равна

$$
M=M_{\mathrm{Si}}+2 M_{\mathrm{O}}=60.06 \mathrm{~g}
$$

с учетом еe составляющих $M_{\mathrm{Si}}=28.086 \mathrm{~g}$ и $M_{\mathrm{O}}=15.99 \mathrm{~g}[16]$.

Тогда из соотношения (2) массовый коэффициент поглощения рентгеновских лучей в плавленом кварце оказывается равным

$$
\left(\frac{\mu}{\rho}\right)_{\mathrm{SiO}_{2}}=35.054 \mathrm{~cm}^{2} / \mathrm{g} .
$$

При величине плотности плавленого кварца $\rho=2.201 \mathrm{~g} / \mathrm{cm}^{3} \quad[17]$ получаем значения линейного коэффициента поглощения рентгеновских лучей $\mu=77.154 \mathrm{~cm}^{-1}$ и практического пробега кванта рентгеновского излучения $\mathrm{Cu} K_{\alpha_{1}} R=1 / \mu=129.6 \mu \mathrm{m}$.

В случае ПВО вдоль направления скользящего падающего пучка рентгеновских лучей кванты рентгеновского излучения проникают только на расстояние $R / 2$, составляющее для плавленого кварца $64.8 \mu \mathrm{m}=6.48 \cdot 10^{-3} \mathrm{~cm}$. Тогда зона выхода $h$ рентгеновских лучей, испытавших ПВО и возбуждающих плазменные колебания в плавленом кварце, рассчитывается по формуле

$$
h=\frac{R}{2} \sin \alpha=6.48 \cdot 10^{-3} \sin \alpha[\mathrm{cm}] .
$$

\section{2. Расчет плотности локализованных электронов и их числа в зоне выхода $h$}

Плотность локализованных электронов $N_{0}$, формирующих ПВО рентгеновских лучей, рассчитывается из энергии плазмонов $E_{P}$ согласно $[18,19]$ как

$$
N_{0}=\frac{m}{4 \pi \hbar e^{2}} E_{P}^{2}
$$

и в системе СИ эта величина определяется соотношениeM

$$
N_{0}=6.52 \cdot 10^{26} E_{P}^{2} \mathrm{~cm}^{-3}
$$

где $E_{P}$ измеряется в электронвольтах.

Однако электроны твердого тела формируют ПВО рентгеновских лучей и плазменные колебания только в объеме $V=S h$ зоны выхода $h$, где $S$ - площадь засветки поверхности образца пучком падающих рентгеновских лучей в режиме ПВО. При величине $S=2 \mathrm{~cm} \cdot 0.8 \mathrm{~cm}=1.6 \mathrm{~cm}^{2}$ число локализованных электронов $N$, участвующих в формировании ПВО рентгеновских лучей и в возбуждении плазменных колебаний, определится для плавленого кварца соотношением

$$
N=N_{0} V=6.76 \cdot 10^{24} E_{P}^{2} \sin \alpha .
$$

\section{3. Энергия плазмонов $E_{P}$}

Энергия плазмонов $E_{P}$, согласно [14] определяется соотношением

$$
E_{P}=E \sin \alpha,
$$

где $E$ - энергия падающих квантов, составляющая в случае рентгеновского излучения $\mathrm{Cu} K_{\alpha_{1}} 8048 \mathrm{eV}$. Отсюда практическая расчетная формула для энергии плазмонов имеет вид

$$
E_{P}=8048 \sin \alpha
$$

\section{3. Экспериментальные результаты и их обсуждение}

Рассмотрим особенности ПВО рентгеновских лучей в стеклообразных материалах.

Наблюдаемое на дифрактограмме в случае аморфного плавленого кварца гало при $2 \vartheta=21.5^{\circ}$ (рис. 1) соответствует интерференции рентгеновских лучей, обусловленной парами атомов кремния и кислорода, расстояние между которыми, вычисленное по формуле Керзома-Смита [20], составляет $5.07 \AA$.

В табл. 1 приведены экспериментальные данные и результаты расчетов энергии плазмонов $E_{P}$ и микроскопических характеристик $h$ и $N$ для 16-ти значений азимутального угла $\beta$, что позволяет рассчитать дисперсию плазмонов, т.е. зависимость энергии плазмонов от волнового вектора $\mathbf{k}$ (рис. 2), имеющую вид замкнутой петли с большой площадью (area). В пределах этой

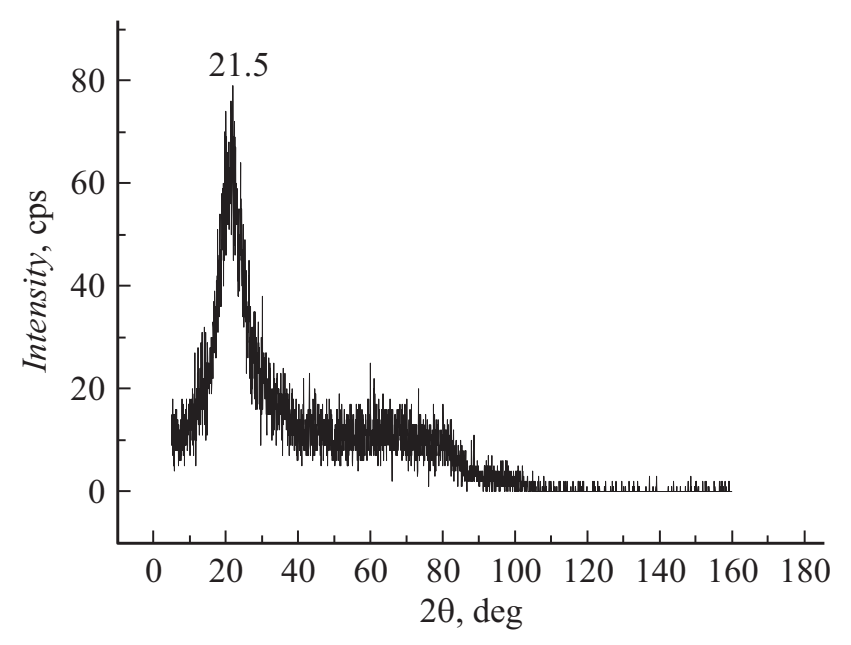

Pис. 1. Дифрактограмма плавленого кварца. 


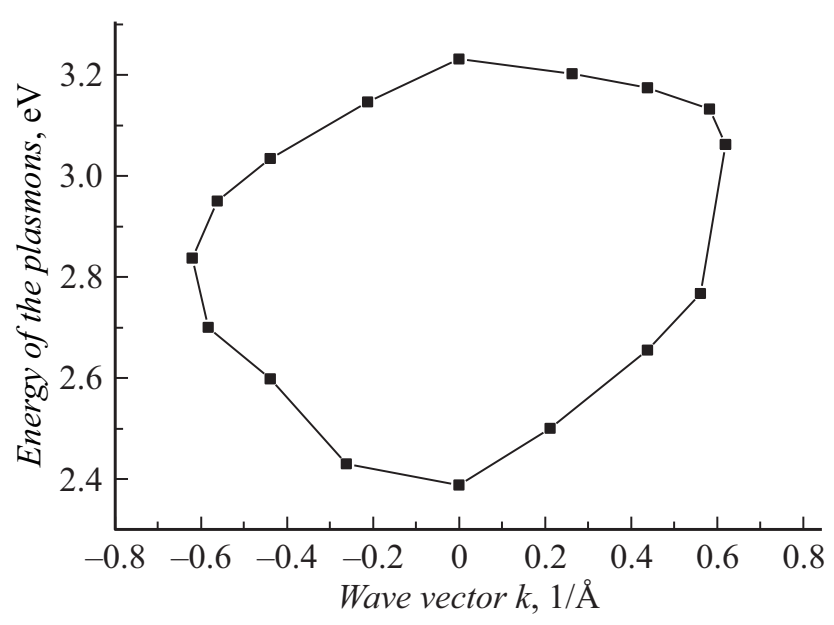

Рис. 2. Кривая дисперсии для плавленого кварца.

Таблица 1. Экспериментальные данные по ПВО и результаты расчетов для плавленого кварца

\begin{tabular}{|c|c|c|c|c|c|}
\hline$\beta^{\circ}$ & $\alpha^{\circ}$ & $E_{P}, \mathrm{eV}$ & $h, \AA$ & $N \cdot 10^{21}$ electrons & $\begin{array}{l}\text { Асимметрия } \\
\text { электронов }\end{array}$ \\
\hline 0 & 0.0218 & 3.062 & 246 & 24 & \multirow{3}{*}{$\frac{N_{0}}{N_{180}}=1.26$} \\
\hline 22.5 & 0.0223 & 3.132 & 252 & 26 & \\
\hline 45 & 0.0226 & 3.174 & 256 & 27 & \\
\hline 67.5 & 0.0228 & 3.202 & 258 & 28 & \multirow[t]{2}{*}{$\frac{N_{45}}{N_{225}}=1.8$} \\
\hline 90 & 0.023 & 3.231 & 260 & 28 & \\
\hline 112.5 & 0.0224 & 3.146 & 253 & 26 & \multirow{3}{*}{$\frac{N_{90}}{N_{270}}=2.54$} \\
\hline 135 & 0.0216 & 3.034 & 244 & 23 & \\
\hline 157.5 & 0.021 & 2.95 & 238 & 22 & \\
\hline 180 & 0.0202 & 2.837 & 228 & 19 & \multirow{8}{*}{$\frac{N_{135}}{N_{315}}=1.44$} \\
\hline 202.5 & 0.0192 & 2.7 & 217 & 16 & \\
\hline 225 & 0.0185 & 2.598 & 209 & 15 & \\
\hline 247.5 & 0.0173 & 2.43 & 196 & 12 & \\
\hline 270 & 0.017 & 2.388 & 192 & 11 & \\
\hline 292.5 & 0.0178 & 2.5 & 201 & 13 & \\
\hline 315 & 0.0189 & 2.655 & 214 & 16 & \\
\hline 337.5 & 0.0197 & 2.767 & 223 & 18 & \\
\hline
\end{tabular}

площади можно вычислить среднюю энергию плазмонов $\bar{E}_{P}($ area $)$, пользуясь соотношением

$$
\bar{E}_{P}(\text { area })=\frac{\sum_{i=1}^{n} E_{P i}}{n},
$$

где $n=30$ - число шагов по оси волнового вектора.
Таким способом в случае плавленого кварца получаем величину средней энергии плазмонов $\bar{E}_{P}($ area $)=0.574 \mathrm{eV}$.

Дополнительной характеристикой дисперсионной кривой является отношение максимальной энергии плазмона $E_{\max }$ к его минимальной энергии $E_{\min }$. В рассматриваемом случае это отношение, определенное по дисперсионной кривой (рис. 2), составило: $E_{\max } / E_{\min }=1.34$. Кроме того, определенный интерес представляет асимметрия числа $N$ локализованных электронов в пределах слоя толщиной $h$, характеризующая степень поляризуемости исследуемого диэлектрика и выражающаяся отношением числа $N$ в диаметрально противоположных направлениях в плоскости образца. В последнем столбце табл. 1 указаны значения асимметрий в четырех диаметрально противоположных направлениях в плоскости образца. Сумма этих асимметрий ( $\sum$ asym) для плавленого кварца составляет 7.04, а средняя асимметрия $(\overline{\operatorname{asym}})-1.76$.

В табл. 2 для стеклообразных диэлектриков приведены микроскопические характеристики при азимутальном угле $\beta=0^{\circ}$, результаты обработки дисперсионных замкнутых кривых и асимметрия локализованных электронов.

Подобно плавленому кварцу все стекла характеризуются большими значениями средней энергии плазмонов, характерными для замкнутых петель и, следовательно, большими внутренними механическими напряжениями. Значения средней асимметрии значительно больше единицы (табл. 2), указывая на высокую степень поляризуемости исследованных образцов стекол.

С целью сравнения со стеклообразными диэлектриками исследованы свойства ПВО рентгеновских лучей в стеклокристаллическом ситалле (содержащий 89 mass.\% оксида кремния $\mathrm{SiO}_{2}$ и 11 mass.\% оксида алюминия $\left.\mathrm{Al}_{2} \mathrm{O}_{3}\right)$ и в монокристалле фторида лития LiF. Соответствующие дифрактограммы приведены на рис. 3 и 4. В случае ситалла (рис. 3) регистрируется поликристал-

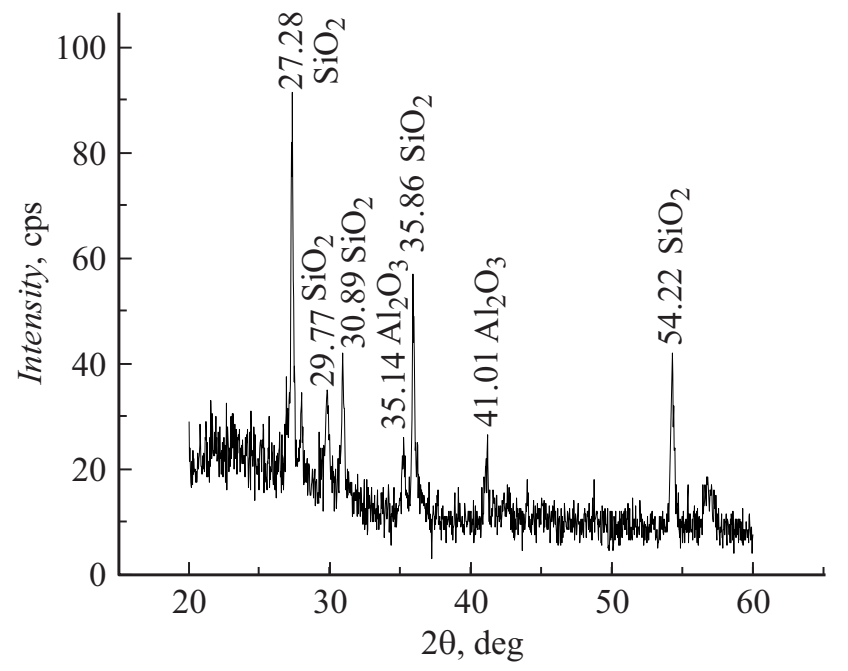

Рис. 3. Дифрактограмма ситалла с указанием над каждым дифракционным пиком значений угла $2 \vartheta$ и вещества. 
Таблица 2. Результаты расчетов и асимметрия для стеклообразных диэлектриков

\begin{tabular}{l|c|c|c|c|c|c|c}
\hline \multicolumn{1}{c|}{ Стекло } & $E_{P}, \mathrm{eV}$ & $h, \AA$ & $N \cdot 10^{21}$ electrons & $\bar{E}_{P}($ area $), \mathrm{eV}$ & $\frac{E_{\max }}{E_{\min }}$ & $\sum$ asym & $(\overline{a s y m})$ \\
\hline Плавленый кварц & 3.062 & 246 & 24 & 0.574 & 1.34 & 7.04 & 1.76 \\
Кварцевое стекло & 3.132 & 252 & 25 & 0.395 & 1.36 & 6.95 & 1.74 \\
Увиолевое стекло & 2.444 & 209 & 13 & 0.412 & 1.428 & 7.85 & 1.96 \\
Молибденовое стекло & 2.725 & 163 & 13 & 0.432 & 1.266 & 6.63 & 1.66
\end{tabular}

Таблица 3. Расчетные характеристики ситалла и монокристалла $\mathrm{LiF}$

\begin{tabular}{l|c|c|c|c|c|c|c}
\hline Вещество & $E_{P}, \mathrm{eV}$ & $h, \AA$ & $N \cdot 10^{21}$ electrons & $\bar{E}_{P}($ area $), \mathrm{eV}$ & $\frac{E_{\max }}{E_{\min }}$ & $\sum$ asym & $(\overline{\text { asym }})$ \\
\hline Ситалл & 3.034 & 202 & 20 & 0.361 & 1.31 & 5.84 & 1.46 \\
$\mathrm{LiF}$ & 3.174 & 619 & 65 & 0.0072 & 1.30 & 6.98 & 1.74
\end{tabular}

лическая орторомбическая структура и большая аморфная стеклообразная фаза, составляющая $80 \%$. В отличие от ситалла дифрактограмма фторида лития (рис. 4) характерна для совершенного монокристалла с кубической структурой, вырезанного по кристаллографической плоскости (200). Соответствующие кривые дисперсии для ситалла и фторида лития приведены на рис. 5 и 6.

Для этих двух исследованных веществ в табл. 3 приведены рассчитанные при азимутальном угле $\beta=0^{\circ}$ микроскопические характеристики, параметры кривых дисперсии плазмонов и асимметрия числа локализованных электронов.

Основным параметром кривой дисперсии плазмонов, отвечающим площади area замкнутой петли (рис. 5 и 6), является средняя энергия плазмонов $\bar{E}_{P}($ area $)$. Стеклокристаллический ситалл по этому параметру близок к аморфным стеклообразным диэлектрикам, в то время как средняя энергия плазмонов монокристалла фторида лития в 80 раз меньше по сравнению с плавленым кварцем. Таким образом, наблюдаемые для аморфных стеклообразных диэлектриков на кривых дисперсии плазмонов замкнутые петли с большой площадью, характери-

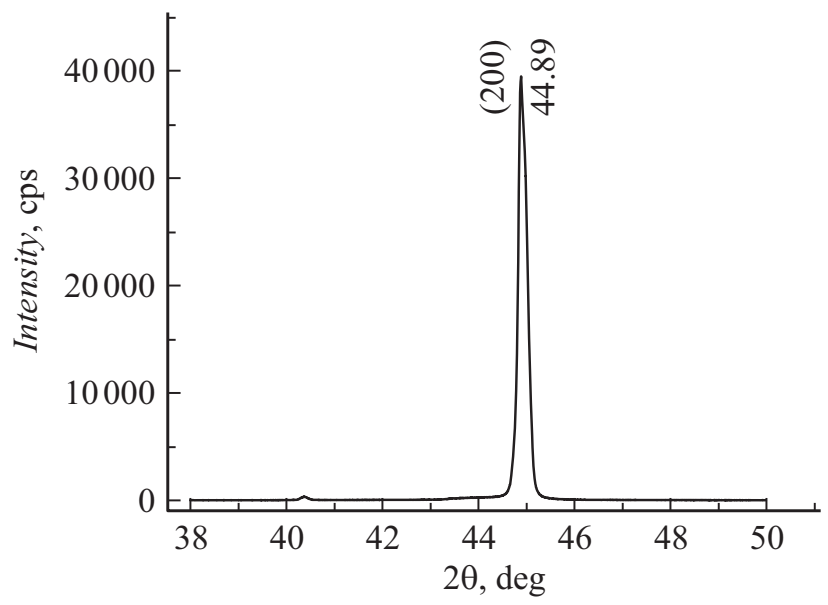

Рис. 4. Дифратограмма монокристалла фторида лития с указанием над дифракционным пиком угла $2 \vartheta$ и индекса Миллера кристаллографической плоскости.

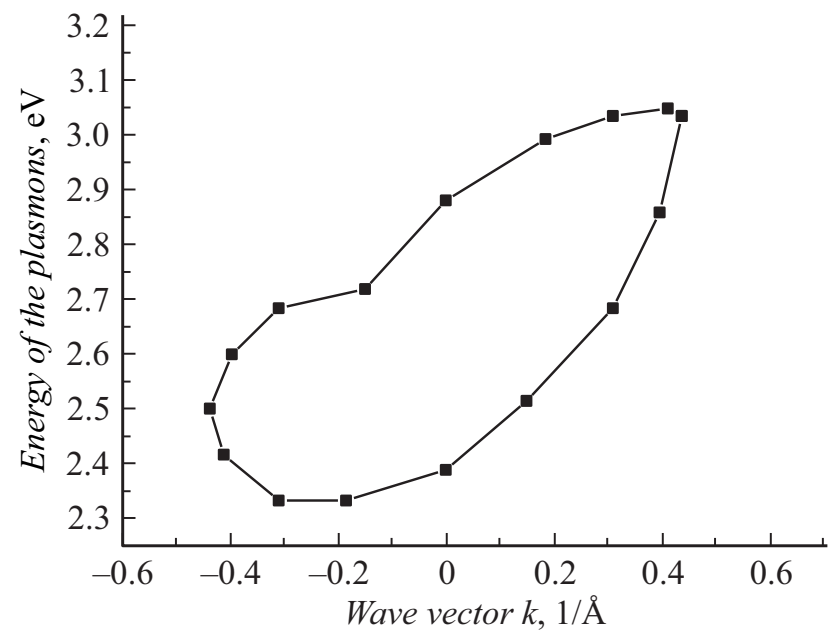

Рис. 5. Кривая дисперсии плазмонов для ситалла.

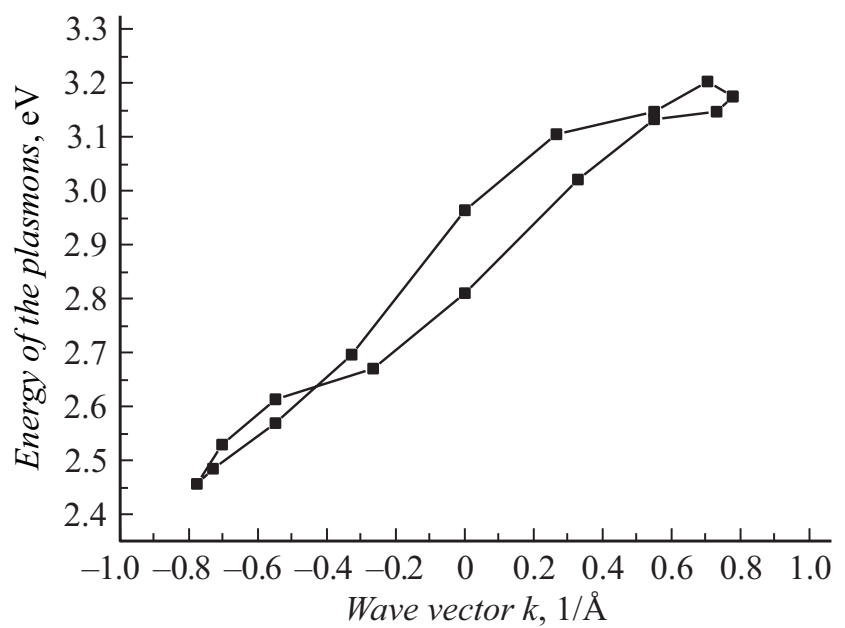

Рис. 6. Кривая дисперсии плазмонов для монокристалла LiF.

зующие высокую среднею энергию плазмонов (табл. 2), свидетельствуют о внутренних механических напряжениях, приводящих к деформации аморфных структур. 
Что касается монокристалла $\mathrm{LiF}$, то отсутствие в нем внутренних напряжений определяет дисперсию плазмонов практически без петли (рис. 6) и соответственно малую среднюю энергию плазмонов. Однако средняя асимметрия для монокристалла $\mathrm{LiF}$ (табл. 3), как и для стеклообразных диэлектриков, намного больше единицы. Это свидетельствует о высокой степени поляризации монокристалла. При этом механизмы поляризации $\mathrm{LiF}$ и стекол разные. Если в стеклообразных диэлектриках поляризация обусловлена деформацией атомов и связанных с ними локализованных электронов, то в монокристалле фторида лития (где практически нет деформации) поляризация является следствием макроскопического дипольного момента кристаллической решетки, образованного молекулами фторида лития с большим дипольным моментом $\mu \sim 6.3 \mathrm{D}$, что на четыре порядка превышает дипольный момент плавленого кварца $\left(\mu \sim 3 \cdot 10^{-4} \mathrm{D}\right)$.

\section{Заключение}

Определение дисперсии плазмонов и асимметрии числа локализованных электронов в зоне $h$ выхода ПВО рентгеновских лучей позволило выяснить механизм поляризации в стеклообразных диэлектриках, связанный с их деформацией. Сравнительное исследование ПВО стеклообразных диэлектриков и монокристалла фторида лития показало иной механизм поляризации монокристалла, связанный с большим макроскопическим дипольным моментом кристаллической решетки LiF. В дальнейшем предполагается исследование методами дисперсии плазмонов и асимметрии числа электронов полупроводников и металлов.

\section{Финансирование работы}

Работа выполнена в рамках государственного задания при финансовой поддержки Министерства науки и высшего образования РФ проект № 3.5005.2017/ВУ.

\section{Конфликт интересов}

Автор заявляет, что у него нет конфликта интересов.

\section{Список литературы}

[1] Фельи А. Аморфные стеклообразные твердые тела. М.: Мир, $1982.545 \mathrm{c}$.

[2] Мотm Н., Дэвис Э. Электронные процессы в некристаллических веществах. М.: Мир, 1982. 350 с.

[3] Брытов И.А., Ромащенко Ю.Н. // ФТТ. 1978. Т. 29. Вып. 6. C. $664-667$.

[4] Брытов И.А., Гриценко В.А. // ЖЭТФ. 1985. Т. 89. С. 562 570.

[5] Некрашевич С.С., Гриценко В.А. // ФТТ. 2014. Т. 58. Вып. 2. С. 209-223.
[6] Волков В.А. Плазмоны и магнитоплазмоны. Влияние на отклик полупроводниковых структур в гига- и терагерцовом диапазоне. М.: ИРЭ РАН, 2019. С. 1-7.

[7] Лисица В.С. Введение в нанооптику. М.: МФТИ, 2012. C. 104-112.

[8] Karalis A., Lidorikis E., Ibanescu M., Joannopoulos J.D. // Phys. Rev. Lett. 2005. PRL95. № 063901. P. 1-4.

[9] Ерёмин М.В., Кочергин Д.С. // Письма в ЖЭТФ. 2018. T. 108. Вып. 2. С. 119-123.

[10] Давидович М.В. // Опт. и спектр. 2019. Т. 126. Вып. 3. C. 360-369.

[11] Baltar H., Drozdowicz-Tomsia K., Goldys F. 2012. USA: Review. P. 10-156.

[12] Стожаров В.М. // ЖТФ. 2017. Т. 87. Вып. 1. С. 125-129.

[13] Стохсаров В.М., Пронин В.П. // ЖТФ. 2017. Т. 87. Вып. 12. C. $1901-1905$.

[14] Стожсаров В.М. // ЖТФ. 2019. Т. 89. Вып. 7. С. 1042-1044.

[15] Блохин М.А., Швейцер И.Е. Рентгеноспектральный справочник. М.: Наука, 1988. С. 374.

[16] Кацнельсон А.А. Рассеяние рентгеновских лучей конденсированными средами. М.: МГУ, 1991. С. 96.

[17] Рабинович В.А., Хавин З.Я. Краткий химический справочник. М.: Химия, 1979. С. 56.

[18] Ашкробт Н., Мермин Н. Физика твердого тела. М.: Мир, 1979. T. 1. 399 c.

[19] Займан Джс. Принципы теории твердого тела. М.: Мир, 1969. 315 c.

[20] Корсунский М.И. Физика рентгеновых лучей. М.-Л.: ОНТИ, 1936 